\title{
Novel AlphasynucleinPAN in human Mesenchymal Stem Cells-derived neurons masks SNCA140 and creates dissociation of deleterious SNCA98 in astrocytoma stem cells
}

\author{
Akeem G. Owoola ${ }^{1-2}$; Rachel K. Okolicsanyi ${ }^{1}$; Lyn R. Griffiths ${ }^{1}$; Larisa M. Haupt ${ }^{1}$ \\ ${ }^{1}$ Genomics Research Centre, Institute of Health and Biomedical Innovation, School of Biomedical Sciences, Queensland University \\ of Technology, 60 Musk Avenue, Kelvin Grove, QLD 4059, Australia \\ ${ }^{2}$ Tissue Repair and Translational Physiology Program, Institute of Health and Biomedical Innovation, School of Biomedical \\ Sciences, Queensland University of Technology, 60 Musk Avenue, Kelvin Grove, QLD 4059, Australia
}

\begin{abstract}
Alzheimer's Dementia (AD) and Parkinson's disease Dementia (PDD) are common causes of dementia characterised by misfolded alpha-Synuclein (SNCA) proteins, amyloid precursor proteins (APP), and microtubule associated protein tau (MAPT). Accumulation of alpha synuclein is involved in cancer. SNCA140 increases oxidative stress, and this leads to elevated amyloidogenic APP processing. There is currently no effective / disease-modifying treatment of AD and PDD. Human mesenchymal stem cells (hMSCs) are promising therapeutic candidates for the repair and regeneration of neural cells, but their viable clinical application in AD and PDD treatment requires consideration of epistatic influence of the lineage substitution of SNCA. Objectives - Here, we hypothesised that SNCAPAN exhibit lineage substitution in hMSCS-derived neurons, and it is epistatic to SNCA140 expression. Methodology - we examined phenotypic characteristic of AD and PDD biomarkers in hMSCs-derived neurons at $p+6$ in four biological replicates $(n=4)$ with non-template controls (NTCS, $n=2)$ for each target gene using a Q-PCR. Amplification reactions in NTCSNCA98 and NTCSNCA115 were validated by gel electrophoresis. SNCA98SNCA126 molecular interaction observed was determined using spearman's rank correlation regression, and this was validated by gel electrophoresis of generic PCR $\left(50^{\circ} \mathrm{C}, 52^{\circ} \mathrm{C}, 54^{\circ} \mathrm{C}, 56^{\circ} \mathrm{C}, 58^{\circ} \mathrm{C}\right.$, and $\left.60^{\circ} \mathrm{C}\right)$ with NTC using Universal human reference RNA (UHRR) and astrocytoma stem cells. Expression of each gene was normalised to $18 \mathrm{~S}$, the endogenous control. Data are presented as mean $2^{(-\triangle \Delta C \mathrm{Ct})} \pm \mathrm{SEM}$. Results SNCAPAN exhibits lineage substitution in hMSC-derived neurons, and it is epistatic to SNCA140 expression, a first time viable novel structural remodelling functional innovation in the treatment of $A D$, PDD, and Brain Cancer. In SNCA98 and SNCA115, there were NTC amplification reactions. SNCA98 and SNCA115 expressions are significantly higher compared to APP $(\mathrm{P}=0.002947735 ; \mathrm{P}=1.3411 \mathrm{E}-07), \mathrm{MAPT}$ $(\mathrm{P}=0.00184993 ; \mathrm{P}=5.15474 \mathrm{E}-05)$, SNCAPAN $(\mathrm{P}=0.003579771 ; \mathrm{P}=3.69932 \mathrm{E}-06)$, SNCA112 $(\mathrm{P}=$ $0.006057562 ; \mathrm{P}=9.06788 \mathrm{E}-05)$, and SNCA126 $(\mathrm{P}=0.001054018 ; \mathrm{P}=2.36 \mathrm{E}-05)$ respectively. SNCA98 expression extremely and significantly correlates with SNCA126 expression $(\rho=0.9827 ; P=3.71669 E-05)$. Conclusion - The UHRR of gel electrophoresis of generic PCR containing SNCA126 in astrocytoma stem cells at $50^{\circ} \mathrm{C}$ and $52^{\circ} \mathrm{C}$ confirms the significant SNCA98-SNCA126 relationship as sign epistasis (deleterious SNCA98 enhances beneficial SNCA126) due to synergistic epistatic background created by SNCAPAN, and this validates the epistasis of SNCAPAN to SNCA140.
\end{abstract}

Keywords: AlphasynucleinPAN, lineage, hMSCs, SNCA140, epistasis, Dementia, astrocytoma

\section{Introduction}

Dementia is a deterioration progressive cognitive malfunction characterised by inability to perform daily activities with psychiatric symptoms [1]. AD the most common cause of dementia represents $60-80 \%$ of cases of dementia and is the main cause of morbidity in the developed world [2]. When APP cellular physiological functions neurogenesis, synaptogenesis, and synaptic plasticity fail, APP breakdown 
products misfold and lead to amyloid beta $(A \beta)$ peptide deposition [3]. MAPT encodes Tau protein, which stabilises microtubules, axons and supports cell functions. Tau protein is controlled by phosphorylation and post-translational modifications. In AD brain, higher phosphates aggregate ( 3 times compared with healthy brain) hyperphosphorylates Tau protein, reducing Tau affinity for microtubules and proteases degradation. This leads to Tau fibrillisation and accumulation into neurofibrillary tangles [4]. AD is characterised by $A \beta$ deposition, Tau pathology, and neurodegeneration [5-6].

Alpha-synuclein ( $\alpha$-Syn) is a 140 amino acid protein encoded by the SNCA gene [7]. It is $14.5 \mathrm{kDa}$ encoded by 5 exons with total transcripts length of 3041 bps maps on 4q21.3-q22. Lewy bodies the abnormal $\alpha$ Syn aggregates in neurons [8] can alone cause Dementia with Lewy bodies (DLB) [8] or destroy dopaminergic nerve cells which produces an excitatory neurotransmitter called dopamine, leading to severe neuronal loss in PD. Interestingly, aggregate of Lewy bodies, A $\beta$ plaque, and tau tangles in the cortex results in PDD [8-9], a characteristic feature of AD. $\alpha$-Syn increases oxidative stress, which promotes JNK - or NF-KB - mediated BACE 1 transcription, alter subcellular localisation of BASE 1, and thus increases amyloidogenic APP processing [10-12].

In HEK293T cells, canonical SNCA140 have been reported to exhibit a strong aggregation property compared to the lower aggregation property of alternative spliced minor isoforms SNCA98, SNCA112, and SNCA126 [13]. Higher level of SNCA140 have been reported in AD and PDD patients [14-16]. With therapeutic approaches limited due to adverse side effects and symptom effectiveness, there is currently no effective / disease-modifying treatment of AD and PDD. HMSCs are promising therapeutic candidates for the repair and regeneration of neural cells because of their native expression of significant heparan sulfate proteoglycans core proteins and inherent ability to differentiate towards neural lineages [Rachel Okolicsanyi et al., 2015]. However, their viable clinical application in AD and PDD treatment requires consideration of epistatic influence of the lineage substitution of SNCA. Thus, we hypothesised that SNCAPAN exhibit lineage substitution in hMSCs-derived neurons, is epistatic to SNCA140 expression, and create synergistic epistasis in astrocytoma stem cells.

\section{MATERIALS AND METHODS}

\subsection{Preparation of lysates and Q-PCR}

Cell pellets of three pooled technical replicate from hMSCs-derived neurons were resuspended TRIzol ${ }^{\circledR}$ reagent (cat\# 15596026, Invitrogen) and RNA was extracted using the Direct-zol ${ }^{\mathrm{TM}}$ RNA MiniPrep kit (cat\# 
R2050, Zymo Research) with RNA samples treated in-column with DNase I (cat\# R2050, Zymo Research) as described by the manufacturer. For cDNA conversion, $150 \mathrm{ng}$ of RNA sample was incubated with iScript $^{\mathrm{TM}}$ reverse transcriptase (cat\# 1708891, Bio-rad) according to the manufacturer's instructions. For Q-PCR analysis, we examined phenotypic characteristic of AD and PDD biomarkers in hMSCs-derived neurons at $p+6$ in four biological replicates $(n=4)$ with non-template controls (NTCs, $n=2$ ) for each target gene: independent variable SNCAPAN, dependent variable canonical SNCA140, positive controls alternative spliced minor isoforms (SNCA98, SNCA112, SNCA115, SNCA126), and negative controls APP and MAPT. Each reaction mix contained $120 \mathrm{ng}$ of cDNA, $100 \mathrm{Nm}$ of gene-specific forward and reverse primers, $5 \mu \mathrm{L}$ of SYBR $^{\circledR}$-Green PCR Master Mix, $0.1 \mu \mathrm{L}$ of passive reference (ROX) dye (GoTaq ${ }^{\circledR}$ Q-pcr Master Mix, cat\# A6001, Promega), and $1.1 \mu \mathrm{L}$ of nuclease-free $\mathrm{H}_{2} 0$. Amplification was monitored on a QuantStudio $^{\mathrm{TM}}$-7 (ThermoFisher Scientific) with cycle conditions consisting of enzyme activation (predenaturation) of 2 minutes at $50^{\circ} \mathrm{C}$ and 3 minutes at $95^{\circ} \mathrm{C}$, followed by (denaturation) 50 cycles of 3 seconds at $95^{\circ} \mathrm{C}$, and (Annealing and extension) 30 seconds at $60^{\circ} \mathrm{C}$.

2.2. Validations and statistical analysis

Gel electrophoresis of the q-PCR target genes was conducted to validate the q-PCR amplification reactions in the NTCSNCA98 and NTCSNCA115. In addition, generic PCR of SNCA98 and SNCA126 was conducted to validate the observed molecular interaction between SNCA98 and SNCA126 at $50^{\circ} \mathrm{C}, 52^{\circ} \mathrm{C}, 54^{\circ} \mathrm{C}, 56^{\circ} \mathrm{C}$, $58^{\circ} \mathrm{C}$, and $60^{\circ} \mathrm{C}$ with NTC using Universal human reference RNA (UHRR) and astrocytoma stem cells. This was followed by gel electrophoresis of the generic PCR of the SNCA98 and SNCA126. Expression of each gene was normalised to $18 \mathrm{~S}$, the endogenous control. Data are presented as mean $2^{(-\Delta \Delta C t)} \pm \mathrm{SEM}$. Spearman's rank correlation regression was used to determine the molecular interaction between SNCA98 and SNCA126. Independent student t-test was used to determine relationship between data. Statistical significance was taken as $\mathrm{P}<0.05$.

\section{RESULTS}

3.1. Average relative expression of $A D$ and PDD biomarkers in $h M S C$-derived neurons at $p+6$ in four biological replicates $(n=4)$ with non-template controls (NTCs, $n=2$ ) for each target gene (figure $1 \mathrm{~A})$. 


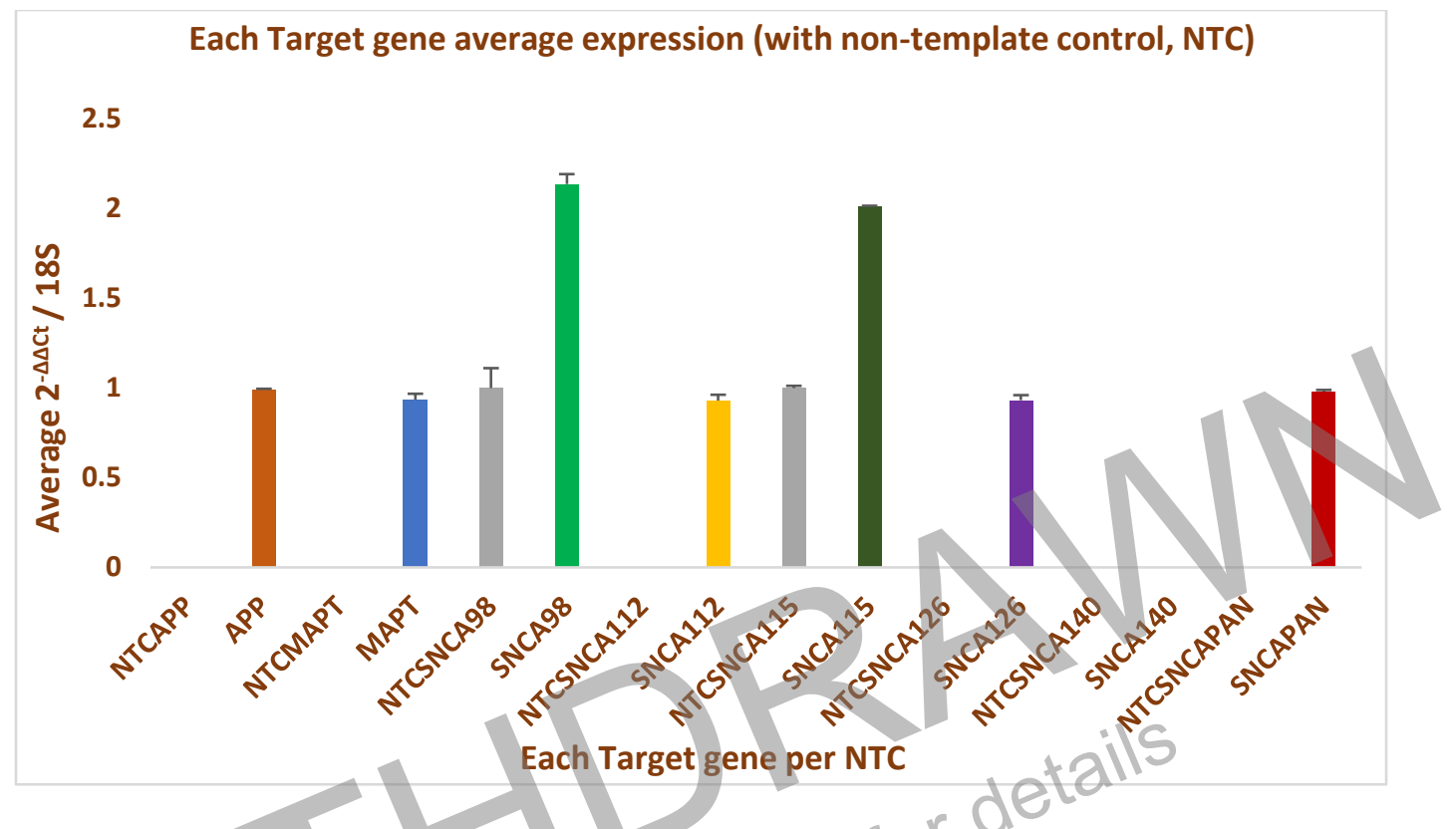

Figure $1 \mathrm{~A}$ - Average relative expression of $A D$ and PDD biomarkers in hMSCs-derived neurons at $p+6$ in four biological replicates $(n=4)$ with non-template controls $(N T C s, n=2)$ for each target gene. There were no amplification reactions in the NTCAPP, NTCMAPT, NTCSNCA112, NTCSNCA140, and NTCSNCAPAN, but there was amplification reaction in NTCSNCA98 and NTCSNCA115. SNCA140 was not expressed due to the epistatic influence of SNCAPAN. Expression of each gene was normalised to $18 \mathrm{~S}$, the endogenous control. Data are presented as mean $2^{\text {(- } \triangle \Delta C \mathrm{Ct})}$ \pm SEM.

To assess epistatic influence of the lineage substitution of SNCA in hMSC-derived neurons, SNCAPAN along with the positive and negative controls was examined via the Q-PCR. There was no amplification reaction in the NTCAPP, NTCMAPT, NTCSNCA112, NTCSNCA140, and NTCSNCAPAN, but there was amplification reaction in NTCSNCA98 and NTCSNCA115 (Figure 1a). In addition, SNCA98 demonstrated highest expression level compared to SNCAPAN, SNCA126, SNCA115, SNCA112, MAPT, and APP (Figure 1a). However, the canonical SNCA140 was not expressed (Figure 1a). Normal human brains have been reported to exhibits lower expression of the alternative spliced minor isoforms SNCA98, SNCA112, and SNCA126 compared to the canonical SNCA140 [13], indicating that SNCAPAN expression masks the expression of the canonical SNCA140 in hMSCs-derived neurons.

Moreover, SNCA98 and SNCA115 expressions are significantly higher compared to APP $(P=0.002947735$; $\mathrm{P}=1.3411 \mathrm{E}-07), \operatorname{MAPT}(\mathrm{P}=0.00184993 ; \mathrm{P}=5.15474 \mathrm{E}-05)$, SNCAPAN $(\mathrm{P}=0.003579771 ; \mathrm{P}=3.69932 \mathrm{E}-06)$, SNCA112 ( $\mathrm{P}=0.006057562 ; \mathrm{P}=9.06788 \mathrm{E}-05)$, and SNCA126 $(\mathrm{P}=0.001054018 ; \mathrm{P}=2.36 \mathrm{E}-05)$ respectively (Figure 1b-c). 


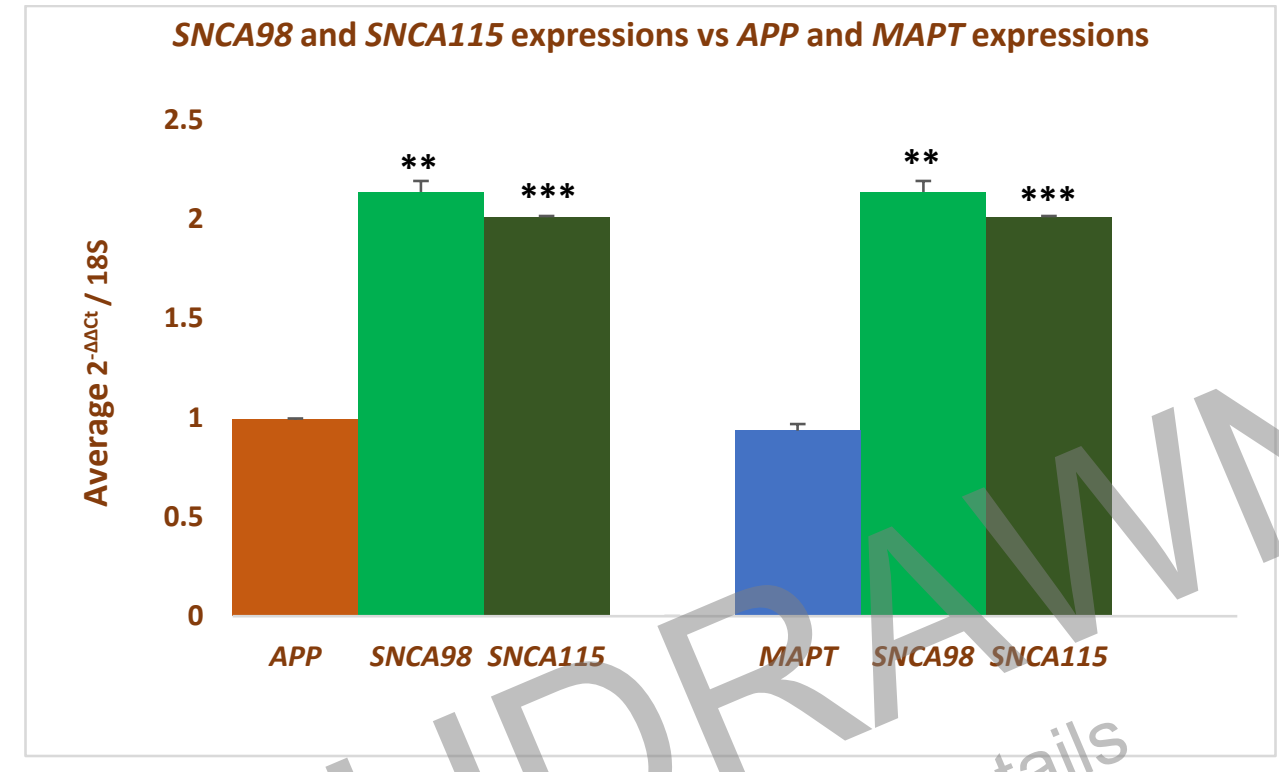

Figure 1B - Average relative expression of SNCA98 and SNCA115 compared with average relative expression of APP and MAPT in hMSCS-derived neurons at $p+6$ in four biological replicates $(n=4)$ with non-template controls (NTCs, $n=2$ ) for each target gene. Brown bar: APP; Blue bar: MAPT; light green: SNCA98; Deep green: SNCA115; *P $<0.005 ; * * \mathrm{P}<0.005 ; * * * \mathrm{P}<0.0001$.

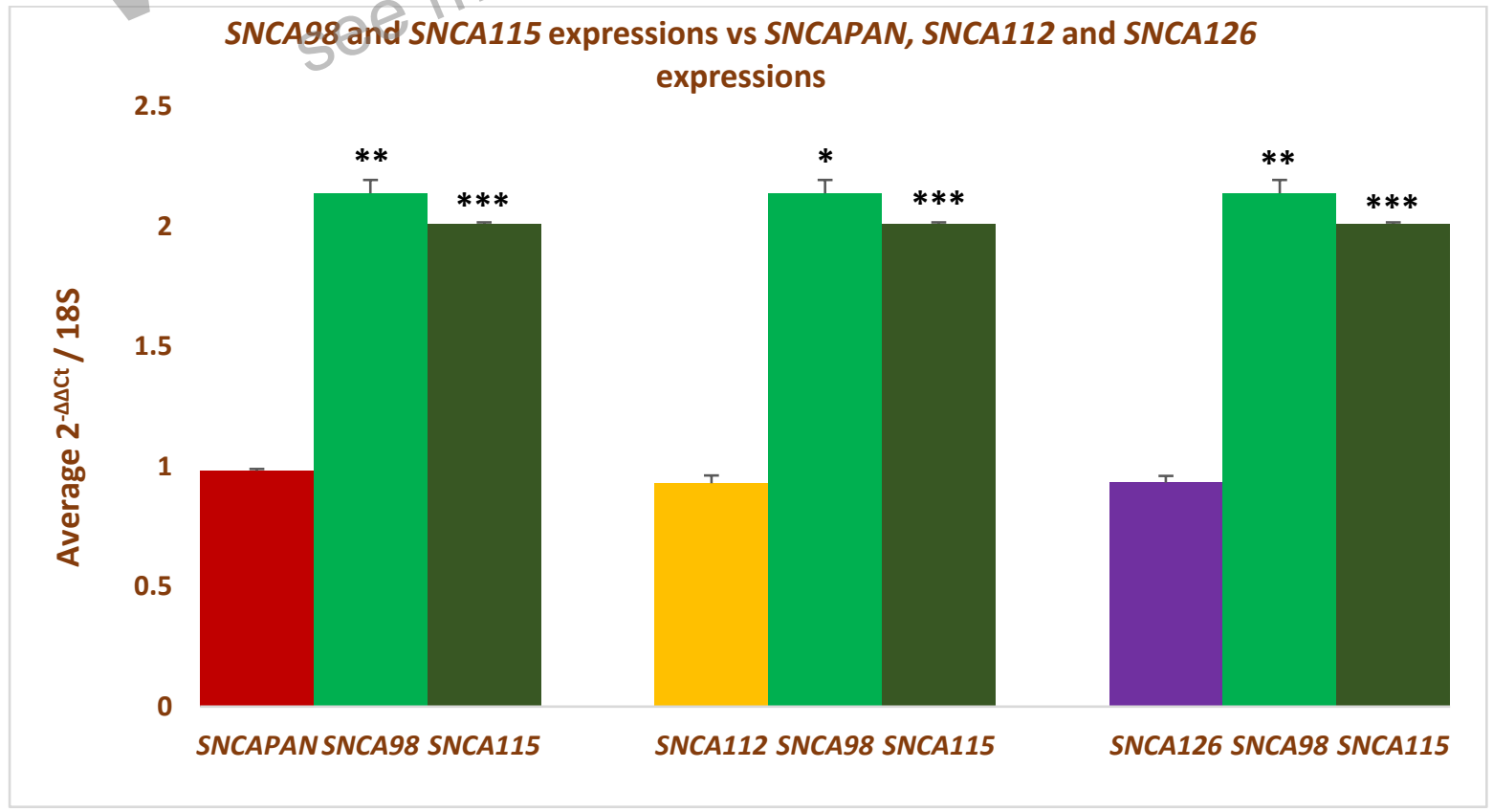

Figure 1C- Average relative expression of SNCA98 and SNCA115 compared with average relative expression of SNCAPAN, SNCA115, and SNCA126 in hMSCs-derived neurons at $\mathrm{p}+6$ in four biological replicates $(n=4)$ with non-template controls (NTCs, $n=2)$ for each target gene. Deep red bar: SNCAPAN; Deep yellow bar: SNCA112; Purple bar: SNCA126; light green: SNCA98; Deep green: SNCA115; * $\mathrm{P}<0.005 ;{ }^{* *} \mathrm{P}<0.005 ;{ }^{* * *} \mathrm{P}<0.0001$. 


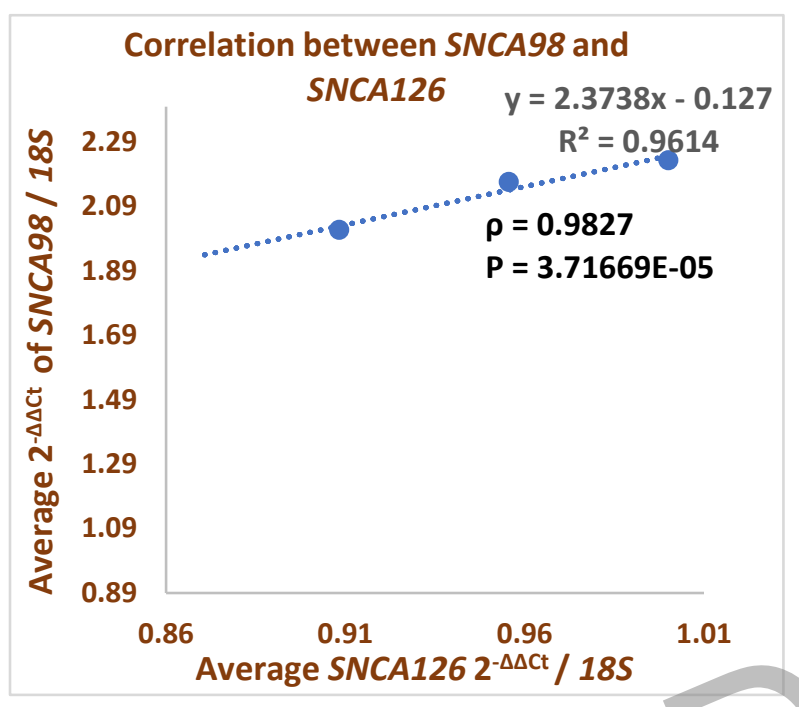

Figure 2B - Scatter Plot showing an extreme high significant positive correlation between average SNCA98 expression and SNCA126 expression in hMSCs-derived neurons at $\mathrm{p}+6$ in four biological replicates $(n=4)$ with nontemplate controls (NTCs, $n=2$ ) for each target gene. $\rho$ : spearman's rank correlation coefficient; $P$ : P-value, significance of the correlation between SNCA98 and SNCA126; $\mathrm{R}^{2}$ : coefficient of determination.

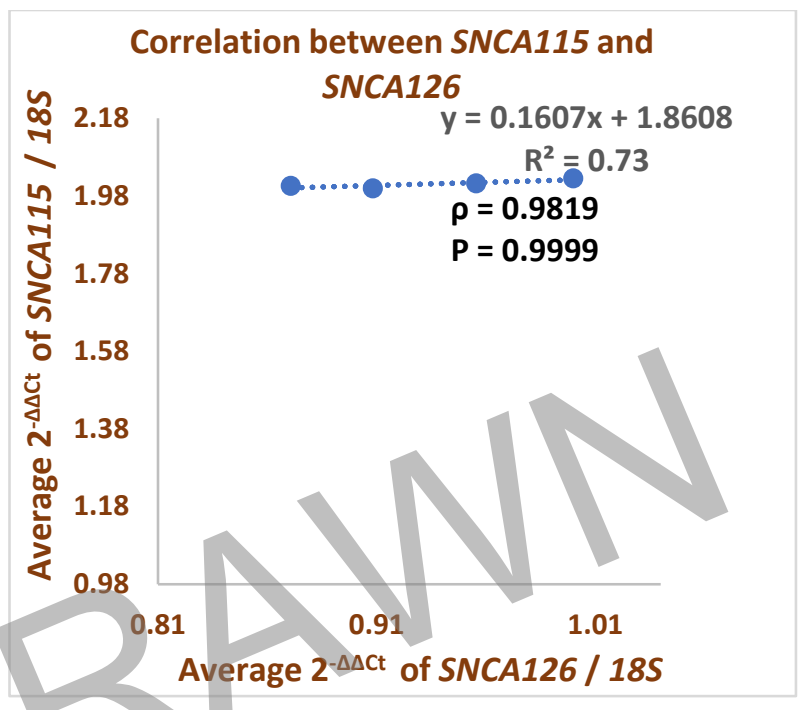

Figure $\mathbf{2 C}$ - Scatter Plot showing a non-significant correlation between average SNCA115 expression and SNCA126 expression in hMSCs-derived neurons at $p+6$ in four biological replicates $(n=4)$ with nontemplate controls (NTCs, $\mathrm{n}=2$ ) for each target gene. $\rho$ : spearman's rank correlation coefficient; $P$ : P-value, significance of the correlation between SNCA115 and SNCA126; $\mathrm{R}^{2}$ : coefficient of determination.

The result shows that SNCA98 expression extremely and significantly correlates with SNCA126 expression $(\rho=0.9827 ; P=3.71669 \mathrm{E}-05)$ (Figure $2 b)$. However, the correlation between SNCA115 and SNCA126 is not statistically significant $(\rho=0.9819 ; P=0.9999)$ (Figure $2 c)$.

We then validated the observed significant molecular interaction between SNCA98 and SNCA126 using gel electrophoresis of the generic $\mathrm{PCR}$ product $\left(50^{\circ} \mathrm{C}, 52^{\circ} \mathrm{C}, 54^{\circ} \mathrm{C}, 56^{\circ} \mathrm{C}, 58^{\circ} \mathrm{C}\right.$, and $\left.60^{\circ} \mathrm{C}\right)$ with $\mathrm{NTC}$, Universal human reference RNA (UHRR), and astrocytoma stem cells (Figure 3 ). 


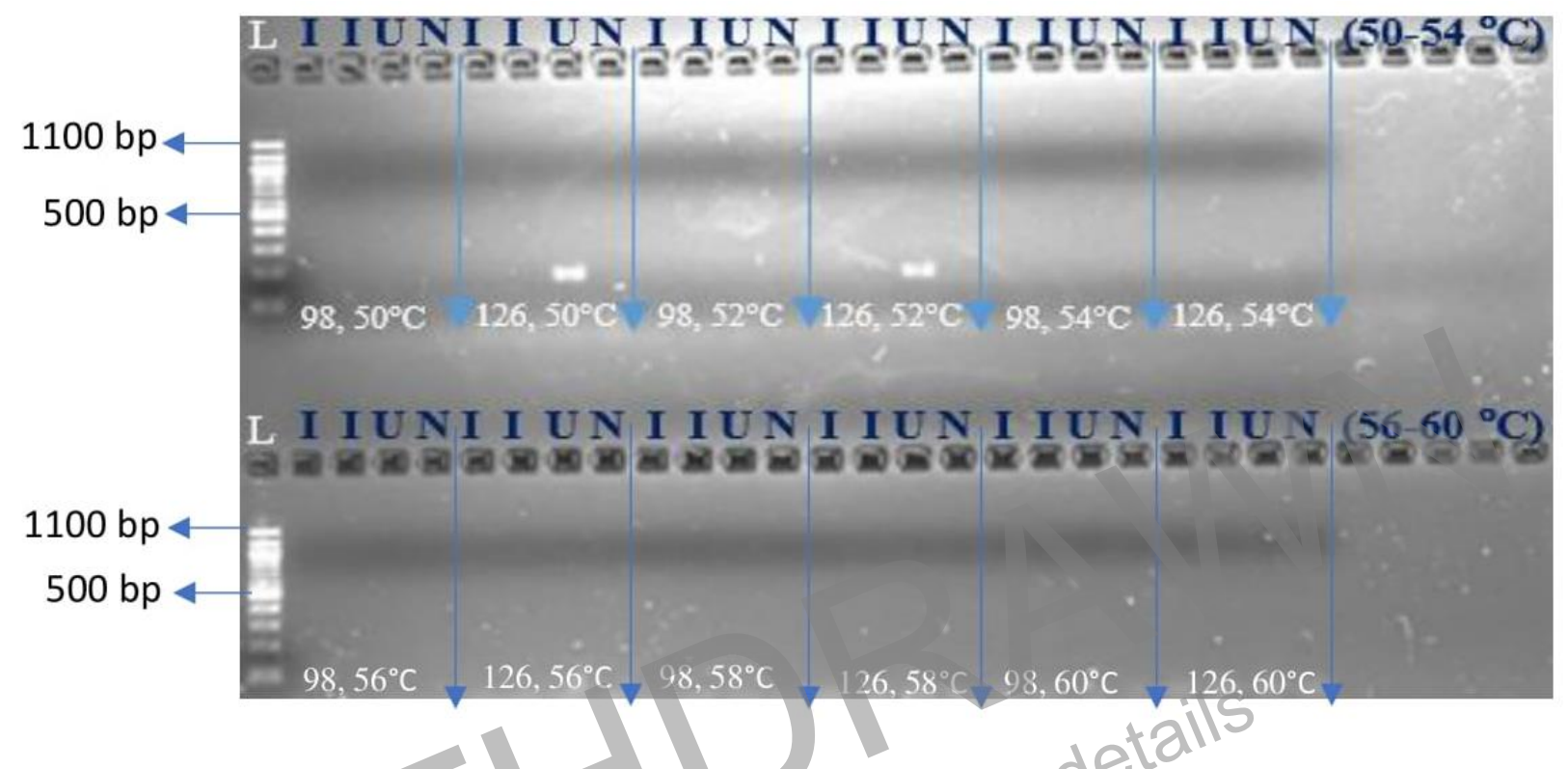

Figure 3: Gel electrophoresis of generic PCR of SNCA98 and SNCA126 exposed to different annealing temperatures (top wells, $50^{\circ} \mathrm{C}-54^{\circ} \mathrm{C}$; bottom wells, $56-60^{\circ} \mathrm{C}$ ). This experiment was conducted to validate SNCA98-SNCA126 molecular interaction. L representS DNA ladder with gel bands of 100 base pairs each. Four wells were used for the gel electrophoresis of the generic PCR per each temperature. For SNCA98 at each temperature, the first-two wells (II) represent astrocytoma stem cells templates which contain SNCA98 each respectively. The third well (U) represents UHRR template which contains SNCA98 and SNCA126. The fourth well( (N) represents the NTC. For SNCA126 at each temperature, the first-two wells (II) represent astrocytoma stem cells templates which contain SNCA126 each respectively. The third well (U) represents UHRR template which contains SNCA98 and SNCA126. The fourth well (N) represents the NTC. Gel electrophoresis of generic PCR product showed that there was no gel band (DNA fragment) in the NTCs across all the temperatures. In addition, there was no gel band in the (a) UHRR template (third well that contains SNCA98 and SNCA126) next to all the astrocytoma stem cells templates (first two wells that contain only SNCA98 each) across all the temperatures (b) UHRR templates (third well that contains SNCA98 and SNCA126) next to all the astrocytoma stem cells templates (first two wells that contain only SNCA126 each) at $54^{\circ} \mathrm{C}, 56^{\circ} \mathrm{C}, 58^{\circ} \mathrm{C}$, and $60^{\circ} \mathrm{C}$. There were gel bands, each representing same sized DNA fragments in the UHRR templates (third well that contains SNCA98 and SNCA126) next to the astrocytoma stem cells templates (first two wells that contain only $S N C A 126$ each) at $50^{\circ} \mathrm{C}$ and $52^{\circ} \mathrm{C} .98$ : SNCA98; 126: SNCA126.

Across all the temperatures, gel electrophoresis of generic PCR product showed that there was no gel band (DNA fragment) in all the NTCs. In addition, there was no gel band in all the UHRR templates (third well that contains SNCA98 and SNCA126) next to all the astrocytoma stem cells templates (first two wells that contain only SNCA98 each) across all the temperatures. Furthermore, there was no gel band in all the UHRR templates (third well that contains SNCA98 and SNCA126) next to all the astrocytoma stem cells templates (first two wells that contain only $S N C A 126$ each) at $54^{\circ} \mathrm{C}, 56^{\circ} \mathrm{C}, 58^{\circ} \mathrm{C}$, and $60^{\circ} \mathrm{C}$. However, there were gel bands, each representing same size DNA fragments in the UHRR templates (third well that 
contains SNCA98 and SNCA126) next to the astrocytoma stem cells templates (first two wells that contain only SNCA126 each) at $50^{\circ} \mathrm{C}$ and $52^{\circ} \mathrm{C}$.

\section{DISCUSSION}

SNCA (encodes a 140 amino acid protein called Alpha-synuclein, $\alpha$-Syn) is one of the main causative genes in neurodegenerative diseases. Lineage specific substitutions occurred during evolution have been mapped on human SNCA with the help of ancestor reconstruction technique [17]. $\alpha$-Syn increases oxidative stress, which promotes JNK - or NF-KB - mediated BACE 1 transcription, alter subcellular localisation of BASE 1, and thus increases amyloidogenic APP processing [10-12]. Oxidative stress is implicated in $A D$ and PDD, and other neurodegenerative diseases. Higher level of intracellular reactive oxygen species (ROS) can cause DNA and protein damage leading to cellular death (). ROS can also trigger mitochondrial and endoplasmic reticulum stress death pathways.

Pan troglodytes (Chimpanzee) SNCA, SNCAPAN, is a neighbor to homo sapiens (human) SNCA in evolutionary relationship of the SNCA family members [17]. Viable clinical application of hMSCs in AD and PDD treatment requires consideration of epistatic influence of the lineage substitution of SNCA. In our study, SNCAPAN exhibits lineage substitution in hMSC-derived neurons, and it is epistatic to SNCA140 expression, a first time viable novel structural remodeling functional innovation in the treatment of $A D$, DLB, PDD, and cancer. Gel electrophoresis of the q-PCR products validated the epistasis of SNCAPAN to SNCA140 expression. According to Bungeroth et al., 2014, higher level of SNCA140 is expressed in normal human brain [13] and Higher level of SNCA140 have been reported in AD and PDD patients [14-16].

Low expression (1\% - 3\% of the canonical SNCA140) of the alternative spliced minor isoforms SNCA126, SNCA112, and SNCA98 have been reported in normal brain [13]. Owing to the amplification reactions in the NTCSNCA98, SNCA98 expressions is significantly highest compared to the expressions of other the positive controls and the negative controls. This is contrary to the lowest expression of SNCA98 in the total brain, Cortex, Nucleus Caudatus, Putamen, and Substantia Nigra [13]. Furthermore, compared to the low total brain expression of SNCA112 in normal brain, the level of SNCA112 in our study is lower. The expression of the remaining positive controls alternative spliced minor isoforms and the negative controls are not consistently higher than each other except for SNCA115. The amplification reactions in the NTCSNCA115 results in a significantly higher SNCA115 expression compared to the other positive controls and the negative controls. 
SNCA115 transcript variants is associated with $\alpha$-Syn protein aggregation. The SNCA115 transcript variant encodes 115-amino acid peptide. However, this transcript variant is highly associated with alcoholism [7]. This could be the reason there is non-significant correlation between average SNCA115 expression and SNCA126 expression in our result. In summary, our investigation opens the door for further structural remodelling functional studies of SNCAPAN in AD, PDD, other neurodegenerative diseases, and cancer.

\section{REFERENCES}

[1] Lin, Y.-T., Wu, P.-H., Kuo, M.-C., Chen, C.-S., Chiu, Y.-W., Yang, Y.-H., . . Chen, H.-C. (2015). Comparison of dementia risk between end stage renal disease patients with hemodialysis and peritoneal dialysis-a population based study. \%J Scientific reports, 5, 8224.

[2] Lin, Y.-T., Wu, P.-H., Kuo, M.-C., Chen, C.-S., Chiu, Y.-W., Yang, Y.-H., . . Chen, H.-C. (2015). Comparison of dementia risk between end stage renal disease patients with hemodialysis and peritoneal dialysis-a population based study. \%J Scientific reports, 5, 8224.

[3] Lei Liu, Li Ding, Matteo Rovere, Michael S Wolfe, Dennis J Selkoe. (2019). A Cellular Complex of BACE1 and $\gamma$-secretase Sequentially Generates $A \beta$ From Its Full-Length Precursor Cell Biol 4;218(2):644663. doi: $10.1083 /$ jcb.201806205.

[4] Newcombe, E. A., Camats-Perna, J., Silva, M. L., Valmas, N., Huat, T. J., \& Medeiros, R. (2018). Inflammation: the link between comorbidities, genetics, and Alzheimer's disease. Journal of neuroinflammation, 15(1), 276.

[5] Spires-Jones, T. L., \& Hyman, B. T. (2014). The intersection of amyloid beta and tau at synapses in Alzheimer's disease. J Neuron, 82(4), 756-771.

[6] Merluzzi, A. P., Vogt, N. M., Norton, D., Jonaitis, E., Clark, L. R., Carlsson, C. M., . . Zetterberg, H. (2019). Differential effects of neurodegeneration biomarkers on subclinical cognitive decline. $J$ Alzheimer's Dementia: Translational Research Clinical Interventions, 5, 129-138.

[7] Cahill, C. M., Aleyadeh, R., Gao, J., Wang, C., Rogers, J. T. (2020). Alpha-Synuclein in Alcohol Use Disorder, Connections with Parkinson's Disease and Potential Therapeutic Role of 5' Untranslated Region-Directed Small Molecules. Biomolecule, 10 (10) 1465.

[8] Alzheimer's Association. (2018). 2018 Alzheimer's disease facts and figures. Alzheimer's Dementia, 14(3), 367-429.

[9] Palermo, G., Tommasini, L., Aghakhanyan, G., Frosini, D., Giuntini, M., Tognoni, G., . . Ceravolo, R. (2019). Clinical Correlates of Cerebral Amyloid Deposition in Parkinson's Disease Dementia: Evidence from a PET Study. Journal of Alzheimer's disease(Preprint), 1-13.

[10] Marwarha G, Raza S, Prasanthi JRP, Ghribi O. Gadd153 and NF-KB crosstalk regulates 27hydroxycholesterol-induced increase in BACE1 and $\beta$-amyloid production in human neuroblastoma SH-SY5Y cells. PLoS One. 2013;8: e70773 10.1371/journal.pone.0070773.

[11] Tan J-L, Li Q-X, Ciccotosto GD, Crouch PJ, Culvenor JG, White AR, et al. Mild oxidative stress induces redistribution of BACE1 in non-apoptotic conditions and promotes the amyloidogenic processing of Alzheimer's disease amyloid precursor protein. PLoS One. 2013;8: e61246 10.1371/journal.pone.0061246.

[12] Roberts, H. L., Schneider, B. L., Brown, D. R. (2017). $\alpha$-Synuclein increases $\beta$-amyloid secretion by promoting $\beta$ - $/ \gamma$-secretase processing of APP. PloS One, 12(2). doi: 10.1371/journal.pone. 0171925.

[13] Bungeroth, M., Appenzeller, S., Regulin, A., Volker, W., Lorenzen, I., Grötzinger, J., Pendziwiat, M., Kuhlenbäumer, G. (2014). Differential aggregation properties of alpha-synuclein isoforms. Neurobiology of Aging, 35 (8), 1913-1919. 
bioRxiv preprint doi: https://doi.org/10.1101/2022.02.08.479501; this version posted February 11, 2022. The copyright holder for this preprint (which was not certified by peer review) is the author/funder, who has granted bioRxiv a license to display the preprint in perpetuity. It is made available under aCC-BY 4.0 International license.

[14] Beyer, K., Domingo-Sàbat, M., Santos, C., Tolosa, E., Ferrer, I., Ariza, A. (2010). The decrease of $\beta$ synuclein in cortical brain areas defines a molecular subgroup of dementia with Lewy bodies. Brain, 133 (12) 3724-3733, https://doi.org/10.1093/brain/awq275.

[15] Beyer, K., Humbert, J., Ferrer, A., Lao, J. I., Carrato, C., Lopez, D., Ferrer, I., Ariza, A. (2006). Low Alphasynuclein 126 mRNA levels in dementia with Lewy bodies and Alzheimer's Disease. Neuroreport, 17 (12), 1327-30.

[16] Recasens, A., \& Dehay B. (2014). Alpha-synuclein spreading in Parkinson's disease. Front Neuroanat 8, doi: 10.3389/fnana.2014.00159.

[17] Siddiqui, I. J. et al. (2016). The Parkinson Disease gene SNCA: Evolutionary and structural insights with pathological implication. Sci. Rep. 6, 24475; doi: 10.1038/srep24475. 


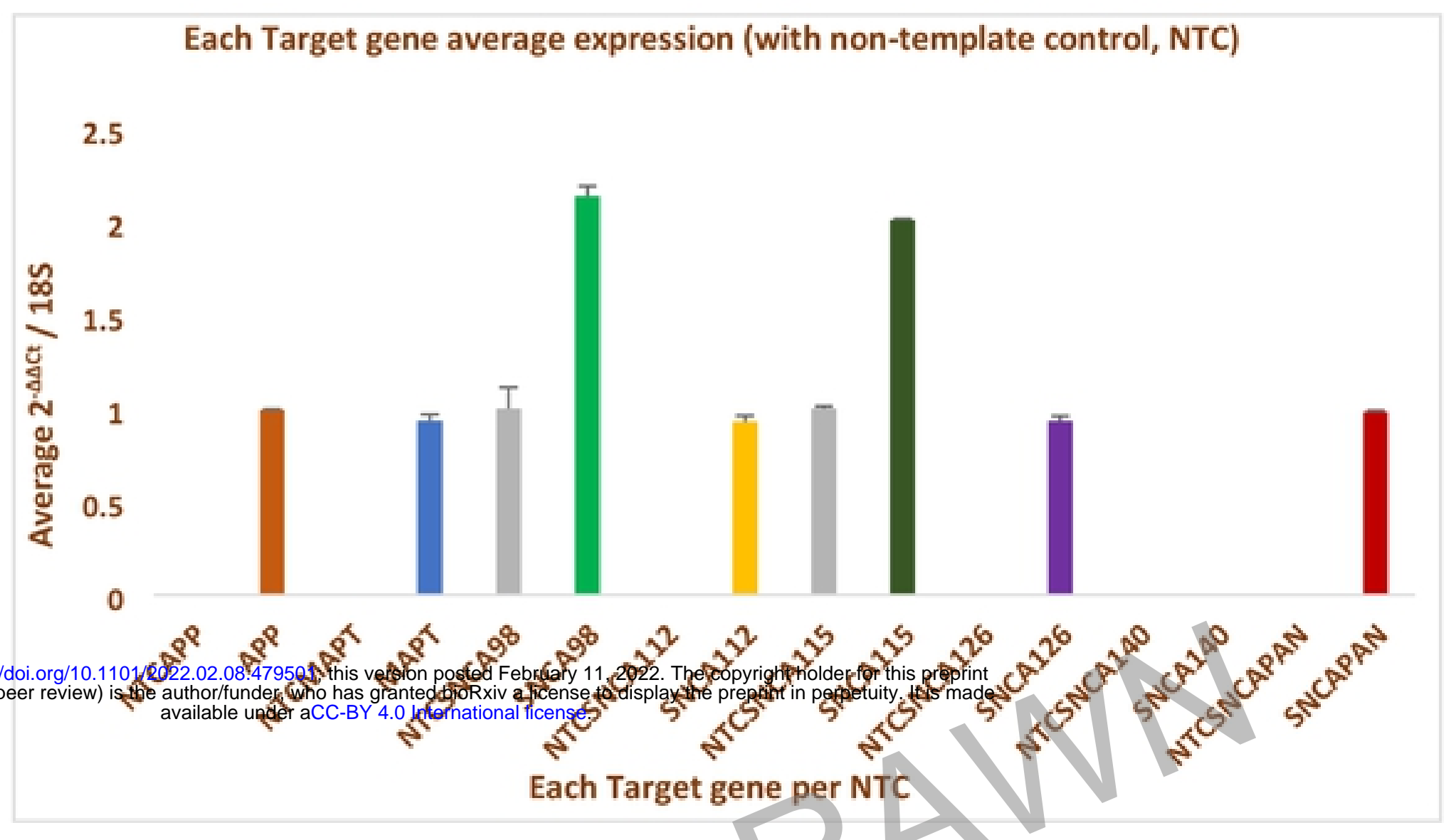

Figure 1A - Average relative expression of AD and PDD biomarkers in $\mathrm{hMSC}$-derived neurons at $p+6$ in four biological replicates $(n=4)$ with non-template controls (NTCs, $n=2$ ) for each target gene. There were no amplification reactions in the NTCAPP, NTCMAPT, NTCSNCA112, NTCSNCA140, and NTCSNCAPAN, but there was amplification reaction in NTCSNCA98 and NTCSNCA115. SNCA140 was not expressed due to the epistatic influence of SNCAPAN. Expression of each gene was normalised to $18 \mathrm{~S}$, the endogenous control. Data are presented as mean $2^{(-\triangle \Delta C t)}$ \pm SEM.

SNCA98 and SNCA115 expressions vs APP and MAPT expressions

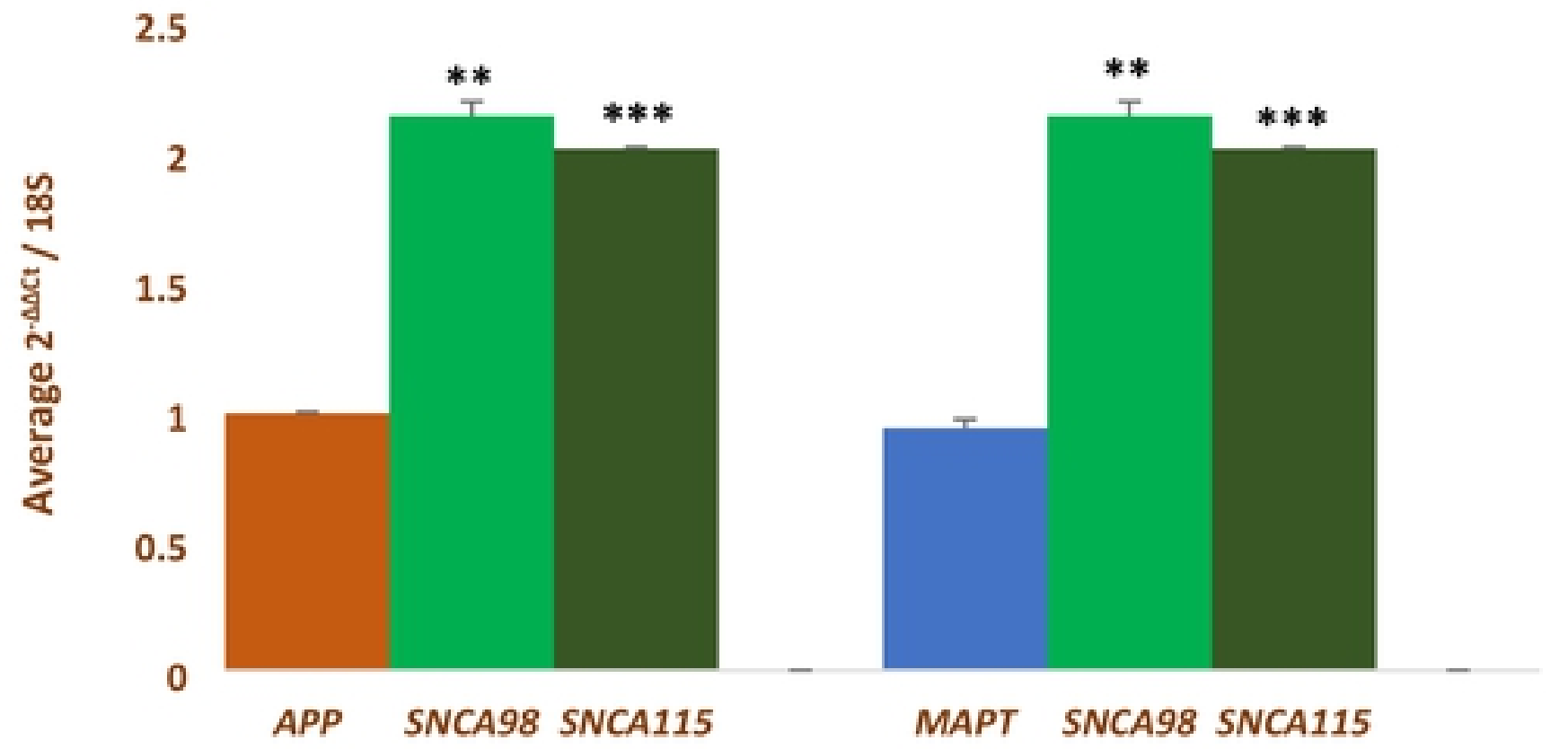

Figure 1B - Average relative expression of SNCA98 and SNCA115 compared with average relative expression of APP and MAPT in hMSCs-derived neurons at $p+6$ in four biological replicates ( $n=4$ ) with non-template controls (NTCs, $n=2$ ) for each target gene. Brown bar: APP; Blue bar: MAPT; light green: SNCA98; Deep green: SNCA115; * $\mathrm{P}<0.005 ;{ }^{* *} \mathrm{P}<0.005 ;{ }^{* * *} \mathrm{P}<0.0001$. 


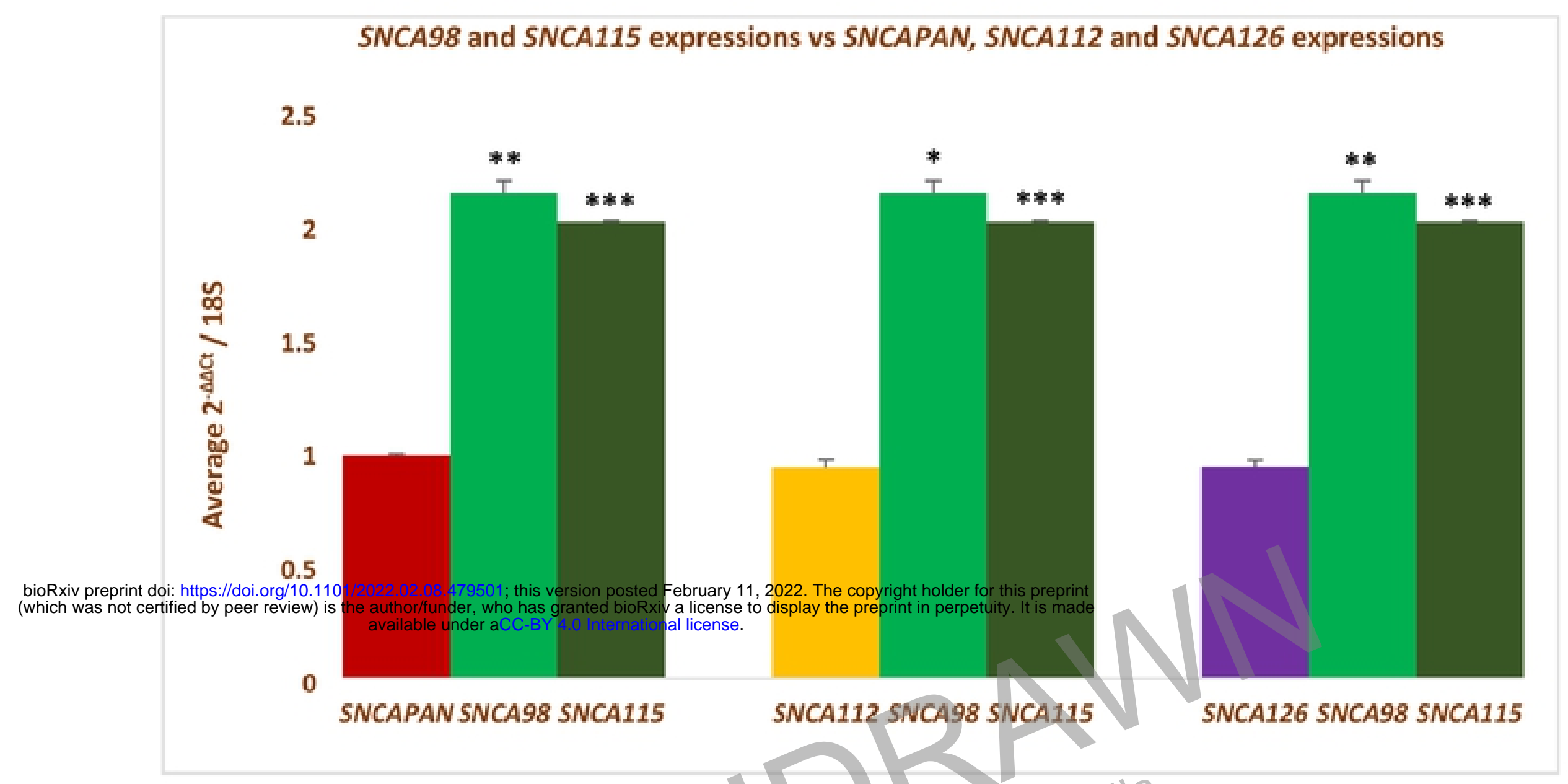

Figure 1C- Average relative expression of SNCA98 and SNCA115 compared with average relative expression of SNCAPAN, SNCA115, and SNCA126 in hMSCS-derived neurons at $\mathrm{p}+6$ in four biological replicates $(n=4)$ with non-template controls (NTCS, $n=2)$ for each target gene. Deep red bar: SNCAPAN; Deep yellow bar: SNCA112; Purple bar: SNCA126; light green: SNCA98; Deep green: SNCA115; * $\mathrm{P}<0.005 ; * * \mathrm{P}<0.005 ; * * * \mathrm{P}<0.0001$.

We validated our q-PCR results using gel electrophoresis (Figure $2 \mathrm{~A}$ ).

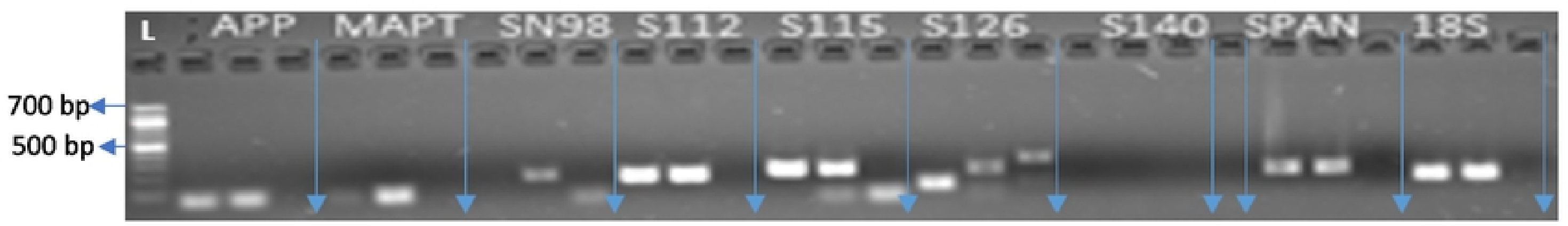

Figure 2A: Gel electrophoresis of the q-PCR target genes using hMSC-derived neurons at $p+6$ as a template. This experiment was conducted to validate q-PCR amplification reactions in the NTCSNCA98 and NTCSNCA115. L represents DNA ladder with gel bands (DNA fragment) of 100 base pairs each. Three wells were used for each gene, including the NTC. The third well represents the NTC for each gene. There was no gel band in (a) the NTCAPP, NTCMAPT, NTCSNCA112, NTCSNCAPAN, NTC18S endogenous control (b) all the wells of SNCA140 (c) the first well of SNCA98. Gel bands observed in the NTCSNCA98 and NTCSNCA115 validated their q-PCR amplification reactions respectively. The NTCSNCA126 gel band was suggested to be a molecular interaction (because there was no amplification reaction in the NTCSNCA126) with either SNCA98 or SNCA115 due to the amplification reactions in the in the NTCSNCA98 and NTCSNCA115. The size of the NTCSNCA126 gel band was higher compared to the size of the NTCSNCA98 gel band and NTCSNCA115 gel band. Furthermore, the intensities of the NTCSNCA98 gel band and SNCA98 second well gel band were reduced compared to the gel bands in SNCA115. As a group, SNCA126 gel bands were not travelling together to the same position compared to the gel bands in the APP, MAPT, SNCA112, SNCA115, SNCAPAN, and the $18 S$. Spearman's rank correlation regression showed that molecular interaction is extremely and significantly between SNCA98 and SNCA126. SN or S: SNCA; 18S: the endogenous control. 

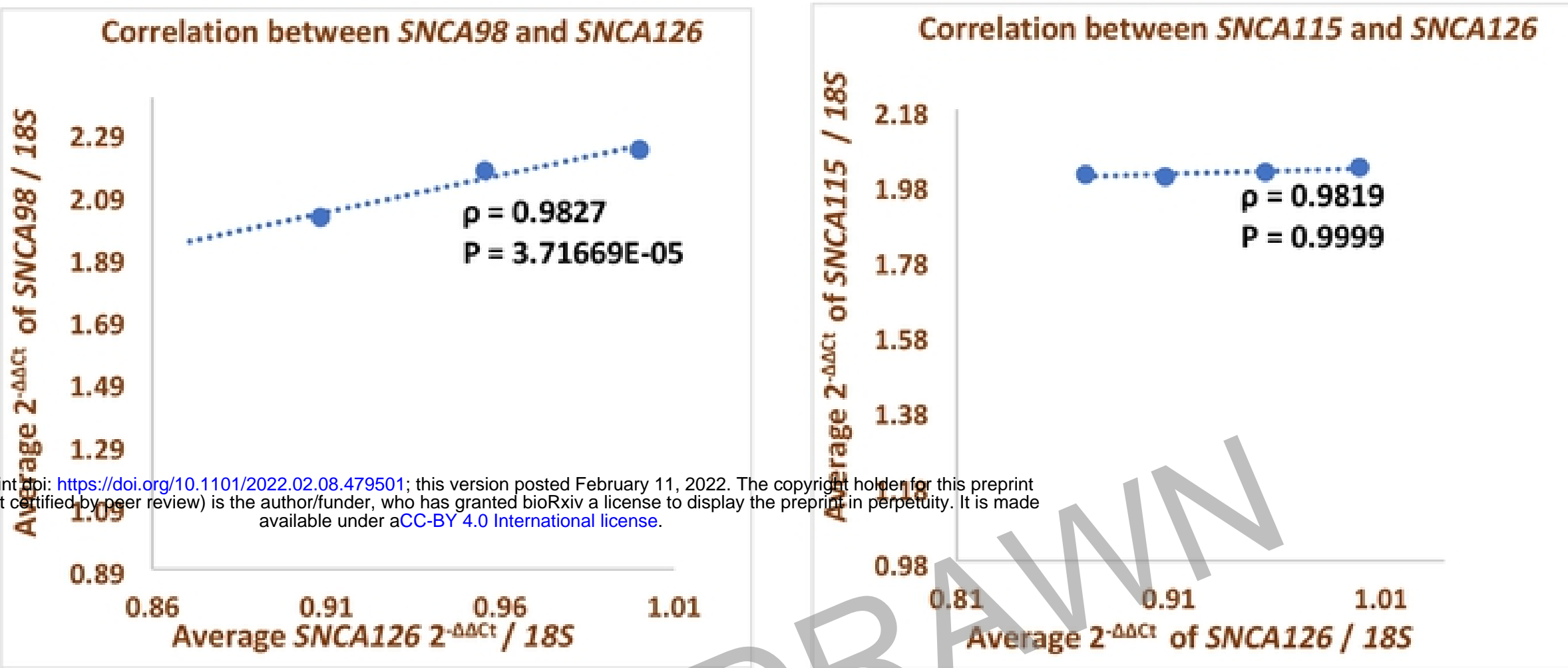

Figure 2B - Scatter Plot showing an extreme high significant positive correlation between Figure 20 -Scatter Plot showing a non-significant correlation between average SNCA115 expression average SNCA98 expression and SNCA126 and SNCA126 expression in hMSCs-derived neurons expression in hMSCs-derived neurons at $p+6$ in four biological replicates ( $n=44$ ) with nontemplate controls (NTCs, $n=2$ ) for each target gene. $\rho$ : spearman's rank correlation coefficient; P: P-value, significance of the correlation between SNCA98 and SNCA126; $\mathrm{R}^{2}$ : coefficient of determination. at $p+6$ in four biological replicates $(n=4)$ with nontemplate controls (NTCs, $n=2$ ) for each target gene. $\rho:$ spearman's rank correlation coefficient; $P$ : P-value, significance of the correlation between SNCA115 and SNCA126; $\mathrm{R}^{2}$ : coefficient of determination. 


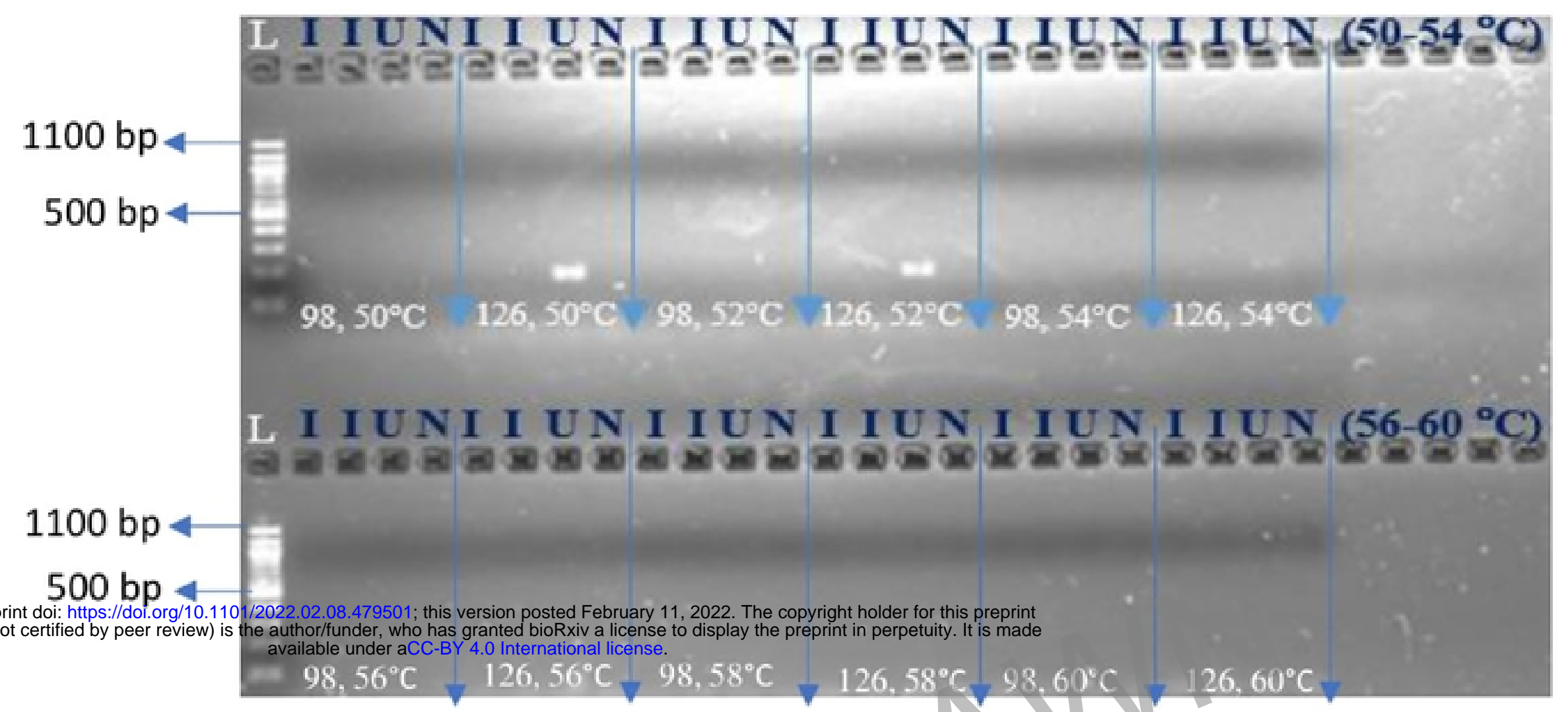

Figure 3: Gel electrophoresis of generic PCR of SNCA98 and SNCA126 exposed to different annealing temperatures (top wells, $50^{\circ} \mathrm{C}-54^{\circ} \mathrm{C}$; bottom wells, $56-60^{\circ} \mathrm{C}$ ). This experiment was conducted to validate SNCA98-SNCA126 molecular interaction. L represents DNA ladder with gel bands of 100 base pairs each. Four wells were used for the gel electrophoresis of the generic PCR per each temperature. For SNCA98 at each temperature, the first-two wells (II) represent astrocytoma stem cells templates which contain SNCA98 each respectively. The third well (U) represents UHRR template which contains SNCA98 and SNCA126. The fourth well (N) represents the NTC. For SNCA126 at each temperature, the first-two wells (II) represent astrocytoma stem cells templates which contain SNCA126 each respectively. The third well (U) represents UHRR template which contains SNCA98 and SNCA126. The fourth well (N) represents the NTC. Gel electrophoresis of generic PCR product showed that there was no gel band (DNA fragment) in the NTCs across all the temperatures. In addition, there was no gel band in the (a) UHRR template (third well that contains SNCA98 and SNCA126) next to all the astrocytoma stem cells templates (first two wells that contain only SNCA98 each) across all the temperatures (b) UHRR templates (third well that contains SNCA98 and SNCA126) next to all the astrocytoma stem cells templates (first two wells that contain only SNCA126 each) at $54^{\circ} \mathrm{C}, 56^{\circ} \mathrm{C}, 58^{\circ} \mathrm{C}$, and $60^{\circ} \mathrm{C}$. There were gel bands, each representing same sized DNA fragments in the UHRR templates (third well that contains SNCA98 and SNCA126) next to the astrocytoma stem cells templates (first two wells that contain only $S N C A 126$ each) at $50^{\circ} \mathrm{C}$ and $52^{\circ} \mathrm{C} .98$ : SNCA98; 126: SNCA126. 